\title{
Providing support to pregnant women and new mothers through moderated WhatsApp groups: a feasibility study
}

\author{
Suha J. Patel ${ }^{1}$, Shalini Subbiah ${ }^{2}$, Rachel Jones ${ }^{2}$, Faith Muigai ${ }^{3}$, Claire Watt Rothschild ${ }^{4}$, Lucille Omwodo ${ }^{2}$, \\ Teresa Ogolla ${ }^{2}$, Grace Kimenju ${ }^{2}$, Nick Pearson ${ }^{2}$, Audra Meadows ${ }^{1}$, Nawal M. Nour ${ }^{1}$ \\ ${ }^{1}$ Division of Global Obstetrics and Gynecology, Brigham and Women's Hospital, Boston, MA, USA; 'Jacaranda Health, Nairobi, Kenya; \\ ${ }^{3}$ PharmAccess Foundation, Nairobi, Kenya; ${ }^{4}$ Department of Epidemiology, University of Washington School of Public Health, Seattle, WA, USA \\ Contributions: (I) Conception and design: SJ Patel, S Subbiah, CW Rothschild, F Muigai, N Pearson, N Nour, A Meadows; (II) Administrative \\ support: N Pearson, F Muigai, N Nour; (III) Provision of study material or patients: SJ Patel, S Subbiah, R Jones, L Omwodo, F Muigai; (IV) \\ Collection and assembly of data: S Subbiah, R Jones, L Omwodo, T Ogolla, G Kimenju; (V) Data analysis and interpretation: SJ Patel, S Subbiah, R \\ Jones, CW Rothschild, N Pearson; (VI) Manuscript writing: All authors; (VII) Final approval of manuscript: All authors. \\ Correspondence to: Suha J. Patel, MD, MPH. Department of Obstetrics and Gynecology, Brigham and Women's Hospital, 75 Francis Street, Boston, \\ MA 02115, USA. Email: sup003@mail.harvard.edu.
}

Background: Group-based health services can improve maternal and newborn health outcomes. Group antenatal care and participatory learning and action cycles (PLA) with women's groups have been cited by the WHO as health systems interventions that can lead to improvements in adherence to care and health outcomes in pregnancy and the postpartum period.

Methods: We used a mixed-methods approach to assess the feasibility of a light touch group-based support intervention using the WhatsApp text-messaging platform. Pregnant women were enrolled at Jacaranda Health (JH), a maternity center in peri-urban Kiambu County, Kenya. Their phone numbers were added to WhatsApp groups consisting of participants with similar estimated due dates. The WhatsApp group administrator was a JH employee. Acceptability, demand, implementation, and practicality of this service were evaluated through in-depth interviews (IDIs), surveys, chart review, and analysis of group chats. Limited analysis of program efficacy (ANC visits, any PNC, and post-partum family planning uptake) was assessed by comparing participant data collected through chart review using a concurrent comparison of the general JH patient population.

Results: Fifty women (88\%) of 57 eligible women who were approached to participate enrolled in the study. Five WhatsApp groups were created. A total of 983 messages were exchanged over 38 weeks. No harms or negative interactions were reported. Participants reported several benefits. Participants had differing expectations of the level of the group administrator's activity in the groups. ANC and PNC attendance were in line with the hospital's metrics for the rest of JH's patient population. Higher rates of postpartum long acting reversible contraception (LARC) uptake were observed among participants relative to the general patient population.

Conclusions: A moderated mobile-based support group service for pregnant women and new mothers is safe and feasible. Additional research using experimental designs to strengthen evidence of the effectiveness of the support intervention is warranted.

Keywords: mHealth; social support; reproductive health; pregnancy; infant care

Received: 13 April 2018; Accepted: 23 April 2018; Published: 21 May 2018.

doi: $10.21037 /$ mhealth.2018.04.05

View this article at: http://dx.doi.org/10.21037/mhealth.2018.04.05 


\section{Introduction}

While rates of maternal and newborn mortality are declining globally, over 300,000 women and 2.6 million newborns continue to die every year from primarily preventable causes $(1,2)$. Empowering and educating women during pregnancy and after birth can have important impacts on care-seeking behaviors and health outcomes $(3,4)$. The $2016 \mathrm{WHO}$ recommendations on antenatal care (ANC) focus on the importance of a positive pregnancy experience for women, and highlight groupbased programs as health systems interventions to improve quality of care and ANC uptake (5). Participatory learning and action cycles (PLA) with women's groups have been found to decrease newborn mortality by approximately one third in low resource settings (6). Group antenatal care services have demonstrated improvements in adherence to care, decreased preterm birth rates in some contexts, and increased satisfaction with care (7). A significant body of evidence links maternal stress to adverse pregnancy outcomes. Women with high self-reported psychosocial stress have a 25-60 percent increased risk of preterm birth as compared to women with low stress (8). Globally, people with self-reported high social support report better health (9). Evidence suggests that social support increases resilience to stress; pregnancy groups that help build social support could therefore plausibly impact health outcomes.

In Kenya, rates of antenatal follow-up are poor with less than 60 percent of pregnant women having at least four antenatal visits (10). Jacaranda Health (JH) operates a maternity center with a mission to provide affordable, high quality care in low-resource settings. The organization is an innovation hub for interventions that improve maternal and newborn health. JH's 18-bed hospital is located on the outskirts of Nairobi, Kenya, and largely serves the informal sector. The hospital offers antenatal care, delivery services (vaginal and cesarean section), postnatal care, family planning services and child wellness care.

In-person group ANC at $\mathrm{JH}$ has shown promising improvements in adherence to ANC and family planning uptake postpartum. However, challenges with staffing and scheduling groups have been barriers to scaling up the intervention. We decided to test a lightweight WhatsAppbased version of group support with the aim of improving social support during pregnancy without the time and logistical requirements associated with in-person groupbased ANC. WhatsApp is increasingly being integrated into the public health space to assist public health workers and improve service delivery (11). However, interventions that seek to use this technology to impact patients are rare. WhatsApp use is ubiquitous among $\mathrm{JH}$ clients, with a 2016 internal survey of $100 \mathrm{JH}$ clients finding 100\% report of WhatsApp as a method of communication. Given the ubiquity of WhatsApp use in the JH patient population, WhatsApp was considered a familiar platform to test the feasibility and acceptability of this intervention.

\section{Methods}

This mixed methods study evaluated the feasibility of mobile-based support groups for pregnant women outside of Nairobi, Kenya. The purpose of this pilot health service was to create a low-cost service for social support with the aim of improving health-seeking behaviors among women who presented to $\mathrm{JH}$ for individual antenatal care. We assessed the intervention by using the following indicators from the feasibility study assessment framework proposed by Bowen et al.: acceptability, demand, implementation, practicality, and limited efficacy (12). Table 1 describes these indicators and the evaluation criteria that we used in this study to assess them:

\section{Study population and study design}

Women presenting for antenatal care at Jacaranda health were recruited and enrolled after obtaining informed consent. Women between age 18-40 years who used WhatsApp and reported their gestational age to be less than 30 weeks were considered eligible for inclusion. We estimated that recruiting women with gestational age below 30 weeks would provide sufficient time for participants to interact in the support groups. On enrollment, clients signed a privacy statement where they pledged to respect the privacy of other clients. Women were enrolled between November and December 2016, with follow-up continuing through October 2017.

Each client's phone number was added to a WhatsApp group consisting of women with estimated date of delivery (EDD) within 2 months of other group members. By week 4 of the recruitment period, all participants were in groups. The study coordinator later confirmed the client's self-reported EDD through chart review. The sample size was limited to 50 women, or 5 WhatsApp groups each consisting of 10 women of similar gestational age. The study coordinator served as the group administrator for each WhatsApp group. She had a basic nursing background and 
Table 1 Feasibility study indicators and evaluation criteria

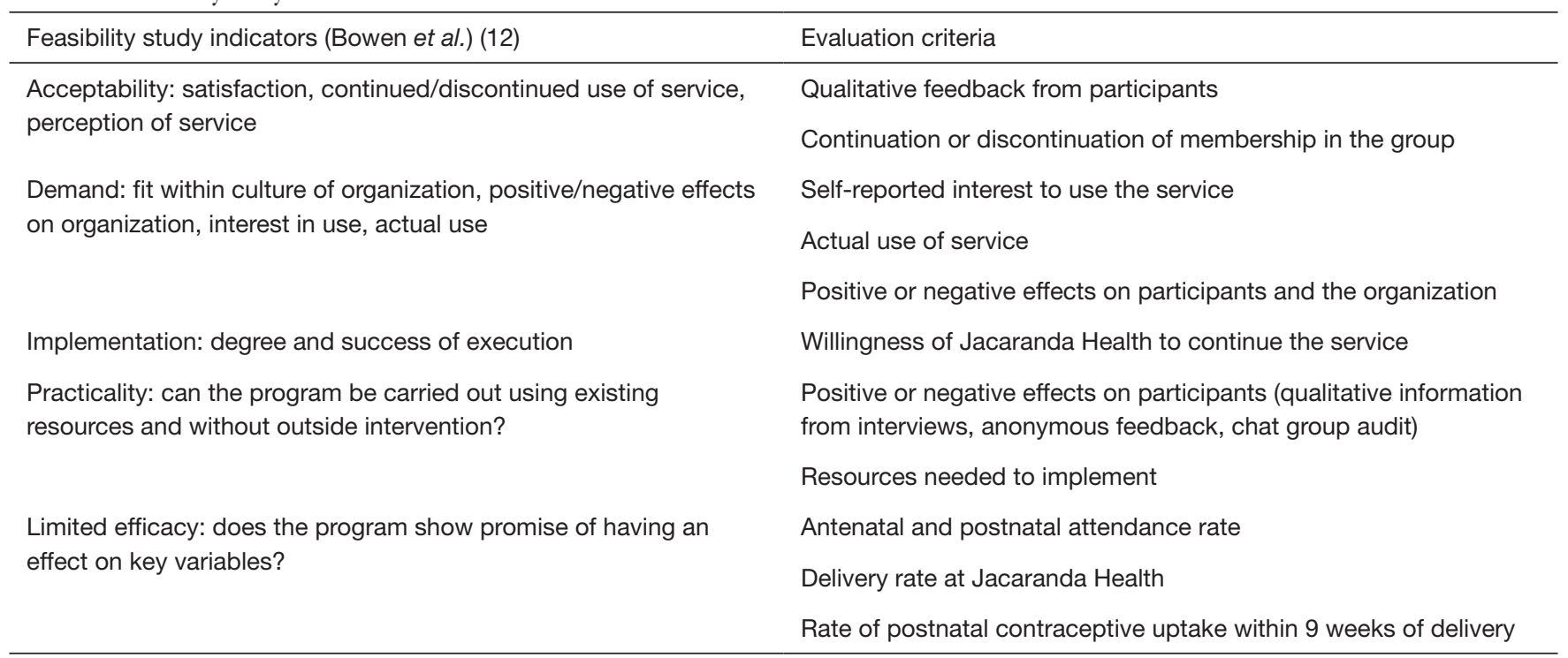

could answer questions regarding care seeking, JH services, and general pregnancy and parenting concerns. A set of guiding question was created, reviewed and approved by $\mathrm{JH}$ clinical leadership. The study coordinator asked questions from this list at her discretion to stimulate conversation in groups. To avoid violations in patient confidentiality, she referred participants to the health facility to address individual medical questions. To encourage respect of group members and confidentiality, participants were required to sign an agreement to a group code of conduct at enrollment.

\section{Data collection}

The study coordinator conducted a weekly audit of each of the chat groups and recorded the following in a log of group activities:

(I) Number of messages exchanged;

(II) Number of participants who exited the groups;

(III) Participant questions and responses;

(IV) Potentially harmful or negative interactions between participants (from the chat group audit and in-depth interviews).

Participants who exited groups before delivery were asked to do an in-depth interview (IDI) over the phone with trained JH research staff. The purpose of this interview was to assess if these women had any negative experiences or barriers to participation. Nine weeks after the delivery of the last participant in the group, participants were sent a link to complete an optional REDCap (13) survey to report any privacy breech or negative experience anonymously. Ten to twelve weeks after delivery, trained research staff conducted IDIs over the phone with two women from each group regarding their experiences in the support groups. Lastly, participants were invited for a group meet-up and focus group discussion facilitated by trained $\mathrm{JH}$ research staff 2-3 months after their estimated dates of delivery. Participants who had not filled out the online survey would have the opportunity to fill it out on paper at this group meet-up.

The study coordinator was not involved with any interviews of study participants so that participants felt that they could speak more freely of their experience in the groups. A professional transcriber was used to transcribe and translate recorded interviews.

Participant demographic information was obtained through chart review. For evaluation of limited efficacy, the following variables were obtainable through routinely collected facility metrics: number of antenatal visits, delivery at $\mathrm{JH}$, postnatal follow-up, and postpartum contraception uptake.

\section{Data analysis}

\section{Qualitative data analysis}

Thematic analysis of IDIs was conducted using grounded theory and open coding in Nvivo 11 (QSR International Pty Ltd). The final list of codes was validated through 
Table 2 Demographics of participants and their WhatsApp groups

\begin{tabular}{|c|c|c|c|c|c|c|c|}
\hline Parameter & Overall, $\mathrm{N}=50$ & Group 1, N=10 & Group 2, N=10 & Group 3, N=10 & Group 4, N=10 & Group 5, N=10 & $P^{*}$ \\
\hline $\begin{array}{l}\text { Median gestational } \\
\text { age [IQR] }\end{array}$ & 21 [17-24] & 13 [8-17] & 18 [14-21] & 20.5 [19-22] & 23 [21-25] & 26 [23-29] & $<0.01$ \\
\hline Nulliparous [\%] & 39 [78] & 9 [90] & 10 [100] & $6[60]$ & $6[60]$ & 8 [80] & 0.12 \\
\hline
\end{tabular}

*, The Kruskal-Wallis test was used to compare median age and gestational age and the Chi Squared test was used to compare parity, history of miscarriage, and marital status.

comparison of coding by a second investigator. Next, codes were categorized into broader groupings. Lastly, the codes and categories were further reduced into general themes. Deductive analysis accompanied the inductive approach above to assess acceptability, demand and practicality of the program's feasibility from the participants' feedback in the IDIs.

\section{Quantitative data analysis}

Quantitative data was analyzed using STATA 14.1 (StataCorp LP, College Station, TX, USA). For group demographic comparisons, the Kruskal-Wallis test was used to compare median age and gestational age and the Chi Squared test was used to compare binary variables (parity, history of miscarriage, marital status) among the five groups.

For limited analysis of efficacy, routinely collected facility data of number of antenatal visits, delivery at $\mathrm{JH}$, postnatal follow-up, postpartum contraception uptake was reported. Rates of delivery at $\mathrm{JH}$, postnatal follow-up and postpartum contraception were compared between the study participants and the rest of the JH ANC population with EDDs in the same time interval as participant deliveries (January to August 2017).

\section{Results}

Fifty pregnant women participated in the study. Sixtyone women presenting for $\mathrm{ANC}$ at $\mathrm{JH}$ were approached for recruitment over the course of 4 weeks. Four of these did not meet study inclusion criteria. Of the 57 who were eligible to enroll in the study, 7 were not interested (12 percent). All enrolled women stated that they were less than 30 weeks although some of these women were uncertain of their gestational age. Upon confirmatory chart review, women were between the gestational age of 6 weeks and
35 weeks at the time of enrollment into the study. Clinical confirmation of gestational age was often different from the patient's self-reported gestational age. Most women had never delivered a baby before and the median gestational age (after confirmation from chart review) was 21 weeks at the time of enrollment (Table 2). Median gestational age varied across groups. Participants were purposefully clustered into groups by similar due dates. Participants delivered between January 2017 to August 2017.

A total of 983 messages were exchanged over 38 weeks. Of these messages, 333 (34 percent) were from the group administrator. Activity varied across groups. Groups one, three and four were more active, whereas groups 2 and 5 were less active (Table 3).

\section{Qualitative findings}

\section{Content analysis}

We conducted a content analysis of questions asked by group participants (excluding the group administrator). A total of 135 questions were asked among all the groups. The most common question topics are listed below in Table 4 as well as the frequencies that questions on these topics occurred among all the groups.

Some other question topics that occurred with less frequency were breastfeeding, fetal movement, headaches, joint pains, leg pains, nose bleeds, due date, post-dates, weight changes, and vomiting in pregnancy.

\section{Interviews}

Research assistants conducted a total of 12 IDIs at 10-12 weeks postpartum. An IDI was administered to at least two participants from each group. There was low participation in focus groups. Three of the group meetings had one participant attend, and none showed up to the other two 
Table 3 Chat group activity

\begin{tabular}{lccccc}
\hline Parameter & Group 1 & Group 2 & Group 3 & Group 4 & Group 5 \\
\hline Total number of messages & 168 & 36 & 209 & 483 & 87 \\
Average number of messages per week & 6 & 1.3 & 7.5 & 18.6 & 3 \\
\hline
\end{tabular}

Table 4 Most common question topics from the WhatsApp groups

\begin{tabular}{lc}
\hline Question topics & Number of questions \\
\hline Cost of services & 11 \\
Postnatal/infant care & 10 \\
JH services and hours & 8 \\
Family planning & 6 \\
Back pain & 6 \\
Ultrasound & 6 \\
Abdominal pain & 5 \\
Preparation for childbirth & 5 \\
Swelling & 4 \\
Insomnia & 4 \\
\hline
\end{tabular}

meetings. An in-person, IDI was conducted with the individuals who came for the scheduled FGD.

Overall, participants reported a positive experience during their participation in the groups. The following themes emerged from qualitative analysis of IDIs.

\section{Informational support}

Informational support was by far the most common benefit described by interviewees. Participants valued the experiences and responses from other women in their groups. Below are some examples:

"My experience was good and learning from other mums I found it easier than my first pregnancy because we used to share and a mother would say something and you find that you are not the only one."

"My experience was good because if you asked any questions they were answered. And we even had members who had experience who were helping us and telling us what to do because obviously in that group we have women who were more experienced than myself and so overall it was good."

\section{Companionship, confidence, and increased health}

\section{seeking}

Other benefits that participants experienced were making new friends, having more confidence as a mother, and encouragement to seek care.
An interviewee indicated expansion of her social network through the groups:

"There was nothing that I didn't like and even another thing that I liked is I made friends and some would save your number and we continued being friends. Even as we speak I met another friend through that group and we have become quite close."

Another interviewee reported stress reduction and empowerment:

"Being a first-time mum it enabled me to be able to take care of my child without worrying. I had that motivation to do all I could as a mum."

An interviewee sought care after prompting from the group:

"I had problems during the nights...I had no comfortable nights and so I asked what to do...I was asked to visit a doctor and when I went there they helped me and they encouraged me and they advised me on what to do..."

\section{Demand}

Interviewees reported that non-participants were interested in the program. A few interviewees also talked about their wishes to continue the program and to bring new women into it. Below are some excerpts from IDIs supporting demand for the program:

"...through that WhatsApp group I have helped around three or four mothers because I was explaining to them that where I go for my clinic we have a WhatsApp group and our admin is a nurse... and they were like I want to go to that hospital; so, I have referred around four people..."

"You can improve [the service] by ensuring it continues to grow so that we keep meeting other women because we keep baving new mothers joining and we belp each other out so I would request that the group continues to be there. And you should not remove the old members but you should instead continue adding more members."

\section{Role of the administrator}

Feedback on the role and activity of the group administrator was conflicting. Four interviewees gave positive feedback about the administrator's activity in the groups. One woman said:

"If you asked the nurse any questions she would respond very fast. For instance, I had this concern about hiccupping and she advised me on what I should do and she also talked to me about 
bygiene during and after delivery."

However, seven interviewees expressed dissatisfaction with the activity and response times of the group administrator. Feedback from three of these women is listed below:

"The facaranda admin was not communicating a lot with the group members so you should improve your interaction and respond quickly to questions when people ask in the group."

"The admin was so slow to some extent answering questions."

"It can be improved if you can add more time... if you are online 24 bours, somebody is there to give us answers..."

\section{Anonymous survey}

Only 7 participants completed the optional, anonymous REDCap survey. All 7 respondents completed it online. None of the respondents reported negative or harmful interactions from participating in the groups.

\section{IDIs with those who exited groups before delivery}

IDIs were conducted with the three women who exited the chat groups before delivery. One of these women had lost her phone and requested to be added back to her group. Another woman reported issues with her phone and trouble accessing WhatsApp although she stated that she wished she could have participated. Only one participant purposefully exited a group. This participant stated that she exited because she was frustrated with the delayed responses from the administrator. This occurred during a time period of three days when the administrator's phone required repairs. However, this participant did say that she had a positive experience overall when she was in the group.

\section{Limited efficacy}

Rates of follow-up and postpartum contraception uptake at JH among study participants were compared to a concurrent sample of the $\mathrm{JH}$ population $(\mathrm{n}=1,106)$ with estimated due dates over the same time interval as participant deliveries (January to August 2017). 62 percent of participants had 4 or more ANC visits at JH. The median number of ANC visits was four as compared to three for the general $\mathrm{JH}$ population $(\mathrm{P}<0.001) .32$ percent of participants delivered at $\mathrm{JH}$ whereas 45 percent of the general $\mathrm{JH}$ antenatal population delivered at $\mathrm{JH}(\mathrm{P}=0.08)$. These comparisons are very limited given that the general $\mathrm{JH}$ population included women who presented for care at the end of the pregnancy with intention to deliver at $\mathrm{JH}$. This difference in the two groups could explain the lower number of ANC visits and the higher delivery rate at $\mathrm{JH}$ among the general JH population. Postpartum follow-up within 9 weeks of delivery at $\mathrm{JH}$ was 30 percent among the participants and 29 percent in the general $\mathrm{JH}$ population $(\mathrm{P}=0.84)$. These women could have received postpartum care at other facilities, but our data was limited to followup at JH. 20 percent of participants took up a postpartum contraceptive method as compared to 15 percent of the general $\mathrm{JH}$ population $(\mathrm{P}=0.38)$. Uptake of long acting reversible contraception (LARC) was 14 percent among participants, which was twice as high as the general $\mathrm{JH}$ population $(\mathrm{P}=0.04)$.

\section{Discussion}

This study evaluates the feasibility of a unique group support service for pregnant and postpartum women in an area with high mobile phone penetration. Overall, this service is feasible in the context of a private, low-cost maternity hospital in Nairobi. Results from this study suggest that mothers have a demand for informational support outside of antenatal visits. The positive perception of the WhatsApp support groups endorses this service's acceptability to participants. No harmful interactions occurred between study participants. Demand was high for this service as seen in the high enrollment rate among eligible women (88 percent). Only one participant purposefully exited her WhatsApp group before delivery. Participants did not report any barriers to access except for two participants who had technical issues with their mobile phones. Results from the qualitative interviews suggest that overall the service was practical for participants. It was also practical for the organization and low-cost. No new or external staff was required for this service. The group administrator was a $\mathrm{JH}$ staff member whose participation in this service was parttime. This employee time was funded by a small grant.

$\mathrm{JH}$ leadership expressed interest in continuing the service since it provides low cost support to mothers outside of ANC visits. However, some areas of improvement were identified to ensure program sustainability. Despite the high number of messages sent from the group administrator, most women reported that the administrator's availability and response time needed improvement. In contrast, JH leadership had concerns regarding time commitment of $\mathrm{JH}$ staff as group administrators in the long term. JH leadership suggested training peer mothers to be group administrators. Another suggestion was to utilize a computerized conversation simulator (Chatbot) to answer general questions promptly. 
A rigorous quantitative evaluation of the intervention's effect on health-seeking behaviors was outside of the scope of this small pilot study. We present comparisons of healthseeking behaviors at $\mathrm{JH}$ between study participants and a concurrent comparison group of 1,106 women seeking antenatal care at Jacaranda Health facilities to provide assessments of the intervention's limited efficacy. A key limitation of this study is the lack of sociodemographic information on the comparison population, which makes it impossible to assess comparability on baseline characteristics to the group who participated in the WhatsApp groups. While we observe that the majority ( $88 \%$ ) of eligible women approached enrolled in the study, it remains possible that women who agreed to participate in the study may be systematically different than Jacaranda's overall antenatal patient population. Future research on this program will involve an RCT to rigorously evaluate the efficacy of this program. The RCT will benefit from the learnings of this study, including improved communication of expectations to participants regarding the group administrator's role and response time. Further investigation is also required to determine if a program like this one is feasible in public and mission hospitals in Kenya since these patient populations may use less mobile data.

\section{Acknowledgements}

Funding: The work presented in this paper was funded by an Expanding the Boundaries Grant from Brigham and Women's Hospital Department of Obstetrics and Gynecology.

The authors would like to acknowledge Catherine Ongeti and Sathy Rajasekharan for their feedback and continued support for this program.

\section{Footnote:}

Conflict of Interest: The authors have no conflicts of interest to declare.

Ethical Statement: The Partners Human Research Committee (USA) and The AMREF Ethics Review Committee (Kenya) reviewed and approved the research. Written informed consent was obtained from all participants.

\section{References}

1. Alkema L, Chou D, Hogan D, et al. United Nations
Maternal Mortality Estimation Inter-Agency Group collaborators and technical advisory group. Global, regional, and national levels and trends in maternal mortality between 1990 and 2015, with scenario-based projections to 2030: a systematic analysis by the UN Maternal Mortality Estimation Inter-Agency Group. Lancet 2016;387:462-74.

2. UNICEF, WHO, World Bank Group and United Nations. Levels and Trends in Child Mortality Report 2017. Oct 2017. Available online: https://www.unicef.org/ publications/index_101071.html

3. Gakidou E, Cowling K, Lozano R, et al. Increased educational attainment and its effect on child mortality in 175 countries between 1970 and 2009: a systematic analysis. Lancet 2010;376:959-74.

4. Karlsen S, Say L, Souza JP, et al. The relationship between maternal education and mortality among women giving birth in health care institutions: analysis of the cross sectional WHO Global Survey on Maternal and Perinatal Health. BMC Public Health 2011;11:606.

5. World Health Organization (WHO). WHO Recommendations on Antenatal Care for a Positive Pregnancy Experience. Geneva: WHO; 2016. Available online: http://apps.who.int/iris/bitstre am/10665/250796/1/9789241549912-eng.pdf?ua=1

6. Tripathy P, Nair N, Barnett S, et al. Effect of a participatory intervention with women's groups on birth outcomes and maternal depression in Jharkhand and Orissa, India: a cluster-randomised controlled trial. Lancet 2010;375:1182-92.

7. Catling CJ, Medley N, Foureur M, et al. Group versus conventional antenatal care for women. Cochrane Database Syst Rev 2015;(2):CD007622.

8. Wadhwa PD, Entringer S, Buss C, et al. The Contribution of Maternal Stress to Preterm Birth: Issues and Considerations. Clin Perinatol 2011;38:351-84.

9. Kumar S, Calvo R, Avendano M, et al. Social support, volunteering and health around the world: crossnational evidence from 139 countries. Soc Sci Med 2012;74:696-706.

10. Keats EC, Ngugi A, Macharia W, et al. Progress and priorities for reproductive, maternal, newborn, and child health in Kenya: a Countdown to 2015 country case study. Lancet Glob Health 2017;5:e782-95.

11. Henry JV, Winters N, Lakati A, et al. Enhancing the supervision of community health workers with WhatsApp mobile messaging: qualitative findings from 2 lowresource settings in Kenya. Glob Health Sci Pract 
2016;4:311-25.

12. Bowen DJ, Kreuter M, Spring B, et al. How we design feasibility studies. Am J Prev Med 2009;36:452-7.

13. Harris PA, Taylor R, Thielke R, et al. Research electronic data capture (REDCap) - a metadata-driven methodology and workflow process for providing translational research informatics support. J Biomed Inform 2009;42:377-81.

doi: 10.21037/mhealth.2018.04.05

Cite this article as: Patel SJ, Subbiah S, Jones R, Muigai F, Rothschild CW, Omwodo L, Ogolla T, Kimenju G, Pearson N, Meadows A, Nour NM. Providing support to pregnant women and new mothers through moderated WhatsApp groups: a feasibility study. mHealth 2018;4:14. 